\title{
Computing free energy differences using conditioned diffusions
}

\author{
Carsten Hartmann and Juan Latorre \\ Institut für Mathematik, Freie Universität Berlin, D-14195 Berlin, Germany
}

\begin{abstract}
We derive a Crooks-Jarzynski-type identity for computing free energy differences between metastable states that is based on nonequilibrium diffusion processes. Furthermore we outline a brief derivation of an infinite-dimensional stochastic partial differential equation that can be used to efficiently generate the ensemble of trajectories connecting the metastable states.
\end{abstract}

Keywords: Conditional free energy, fluctuation theorem, rare events, diffusion bridge

PACS: $02.50 . \mathrm{Ga}, 05.10 . \mathrm{Gg}, 05.70 . \mathrm{Ln}, 65.40 . \mathrm{gh}$

\section{INTRODUCTION}

Given a system assuming states $x \in \mathscr{X} \subseteq \mathbb{R}^{d}$ with the energy $V(x)$, the free energy at temperature $\varepsilon>0$ as a function of a scalar reaction coordinate $\Phi(x)$ is defined as

$$
F(\xi)=-\varepsilon \ln \int_{\mathscr{X}} \exp \left(-\varepsilon^{-1} V(x)\right) \delta(\Phi(x)-\xi) d x .
$$

Given that $x \in \mathscr{X}$ follows the Boltzmann distribution $\rho \propto \exp \left(-\varepsilon^{-1} V\right)$, the free energy is just the marginal distribution in $\Phi(x)$. However estimating the marginal numerically from samples of $\rho$ may be prohibitively expensive, e.g., when $V$ has large barriers in the direction of $\Phi$. Therefore we dismiss this option and propose a different scheme that employs realizations of the overdamped Langevin equation

$$
d X_{\tau}=f\left(X_{\tau}, \tau\right) d \tau+\sqrt{2 \varepsilon} d W_{\tau}, \quad \tau \in[0, T]
$$

subject to the boundary conditions (see Fig. 1)

$$
\Phi\left(X_{0}\right)=\xi_{A} \quad \text { and } \quad \Phi\left(X_{T}\right)=\xi_{B} .
$$

The vector field $f(x, \tau)=-\nabla V(x)+g(x, \tau)$ is assumed to be smooth with the timedependent part $g$ being such that the process hits the level set $\left\{\Phi(x)=\xi_{B}\right\}$ at time $T$; without loss of generality we set $T=1$.

As we will demonstrate below, the free energy difference $\Delta F=F\left(\xi_{B}\right)-F\left(\xi_{A}\right)$ can be computed as the weighted average (cf. $[1,2,3]$ )

$$
\Delta F=-\varepsilon \ln \mathbf{E}\left[\exp \left(-\varepsilon^{-1} \int_{0}^{1} g\left(X_{\tau}, \tau\right) \circ d X_{\tau}\right)\right]
$$

where "o" means integration in the sense of Stratonovich and $\mathbf{E}[\cdot]$ denotes the expectation over all (bridge) paths that solve the conditioned Langevin equation (2)-(3). 


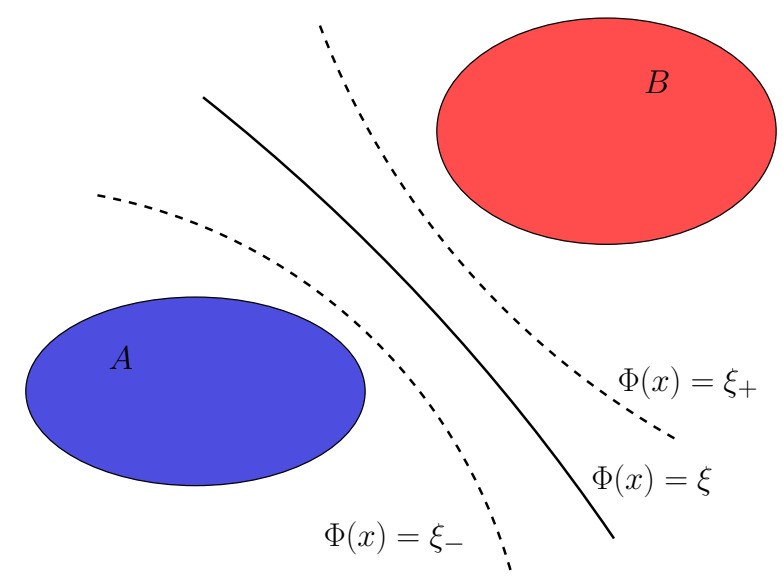

FIGURE 1. Boundaries of metastable states $A$ and $B$ as level sets of the reaction coordinate $\Phi$.

\section{DERIVATION: EULER'S METHOD}

Our derivation of (4) is based on the discrete Euler-Maruyama approximation of (2),

$$
X_{k+1}=X_{k}+\Delta \tau f\left(X_{k}, \tau_{k}\right)+\sqrt{2 \varepsilon \Delta \tau} \eta_{k+1}, \quad k=0, \ldots, n-1 .
$$

Here $\Delta \tau=1 / n$ and $\eta_{k} \sim \mathscr{N}(0, I)$ are i.i.d. distributed Gaussian random variables.

We call $\mathbf{P}_{n}(x)=\operatorname{Prob}\left[X_{0}=x_{0}, X_{1}=x_{1}, \ldots, X_{n}=x_{n}\right]$ the joint distribution of the path $x=\left\{x_{0}, x_{1}, \ldots, x_{n}\right\} \subset \mathscr{X}$. Assuming that the $x_{0}$ follow the Boltzmann distribution $\rho$ conditional on $\Phi\left(x_{0}\right)=\xi_{A}$, the distribution of the paths is readily shown to be

$$
\mathbf{P}_{n}(x) \propto \rho\left(x_{0} \mid \xi_{A}\right) \exp \left(-\frac{\Delta \tau}{4 \varepsilon} \sum_{k=0}^{n-1}\left|\frac{x_{k+1}-x_{k}}{\Delta \tau}-f\left(x_{k}, \tau_{k}\right)\right|^{2}\right) \delta\left(\Phi\left(x_{n}\right)-\xi_{B}\right) .
$$

We are interested in the likelihood ratio of forward and backward paths. To this end we introduce $\tilde{\mathbf{P}}_{n}(x)=\mathbf{P}_{n}(\tilde{x})$ as the distribution of the reversed paths $\tilde{x}=\left\{x_{n}, x_{n-1}, \ldots, x_{0}\right\} \subset$ $\mathscr{X}$ with $x_{n} \sim \rho\left(\cdot \mid \xi_{B}\right)$. By the smoothness of $f$, the forward measure $\mathbf{P}_{n}$ has a density with respect to $\tilde{\mathbf{P}}_{n}$ that is is given in terms of their Radon-Nikodym derivative,

$$
\psi_{n}(x)=\exp \left(\varepsilon^{-1}\left(\Delta V+W_{n}(x)\right)\right) \exp \left(-\varepsilon^{-1} \Delta F\right) .
$$

Here $\Delta V=V\left(x_{n}\right)-V\left(x_{0}\right)$ and

$$
W_{n}(x)=\frac{1}{2} \sum_{k=0}^{n-1}\left(x_{k+1}-x_{k}\right) \cdot\left(f\left(x_{k}, \tau_{k}\right)+f\left(x_{k+1}, \tau_{k+1}\right)\right)+\mathscr{O}(|\Delta \tau|)
$$

is the Stratonovich approximation of the stochastic work integral, i.e.,

$$
\lim _{n \rightarrow \infty} W_{n}(x)=-\Delta V+\int_{0}^{1} g\left(X_{\tau}, \tau\right) \circ d X_{\tau} \quad(\Delta \tau \rightarrow 0, n \Delta \tau=1) .
$$

The free energy difference in (6) pops up as a boundary term, $\exp \left(-\varepsilon^{-1} \Delta F\right)=Z_{B} / Z_{A}$, with $Z_{A}$ and $Z_{B}$ normalizing the conditional distributions for forward and backward paths. Upon noting that both $\mathbf{P}_{n}$ and $\tilde{\mathbf{P}}_{n}$ are probability measures, (6) entails (4) as $n \rightarrow \infty$. 


\section{AN INFINITE-DIMENSIONAL LANGEVIN SAMPLER}

Now comes our main result: To evaluate the expectation in (4) we have to generate the ensemble of bridge paths. For this purpose we introduce the auxiliary potential

$$
\varphi=\Delta \tau^{-1} V\left(x_{0}\right)+\frac{1}{4} \sum_{k=0}^{n-1}\left|\frac{x_{k+1}-x_{k}}{\Delta \tau}+f\left(x_{k}, \tau_{k}\right)\right|^{2}+\Delta \tau^{-1} \varepsilon\left(\ln \left|\nabla \Phi\left(x_{0}\right)\right|+\ln \left|\nabla \Phi\left(x_{n}\right)\right|\right),
$$

so that $\exp \left(-\varepsilon^{-1} \Delta \tau \varphi\right)$ is the density of $\mathbf{P}_{n}$ with respect to the surface element on the image space $\Sigma=\left\{x \in \mathscr{X}^{n+1}: \Phi\left(x_{0}\right)=\xi_{A}, \Phi\left(x_{n}\right)=\xi_{B}\right\} \subset \mathscr{X}^{n+1}$ of admissible paths. Conversely, $\exp \left(-\varepsilon^{-1} \Delta \tau \varphi\right)$ is the stationary distribution of the Langevin equation [4]

$$
d Q_{s}=-\left(\nabla \varphi\left(Q_{s}\right)+\nabla \sigma\left(Q_{s}\right) \lambda^{T}\right) d s+\sqrt{2 \varepsilon \Delta \tau^{-1}} d W_{s}, \quad \sigma\left(Q_{s}\right)=0
$$

where $Q_{s}=\left(q_{0}(s), \ldots, q_{n}(s)\right)$ and $\lambda=\left(\lambda_{1}, \lambda_{2}\right)$ labels the Lagrange multipliers determined by the constraint $\sigma=0$, the latter being shorthand for $\Phi\left(q_{0}\right)=\xi_{A}$ and $\Phi\left(q_{n}\right)=\xi_{B}$.

Using formal arguments (that can be made rigorous using Girsanov's theorem), we can take the limit $n \rightarrow \infty$ which turns the Langevin sampler (7) into a stochastic partial differential equation (SPDE) for bridge paths [5]. If we denote the continuous path by $\gamma=\gamma(\tau, s)$ with $\tau \in[0,1]$ now being the "spatial" variable, our SPDE reads

$$
\begin{array}{rr}
\frac{\partial \gamma}{\partial s}=\frac{1}{2} \frac{\partial^{2} \gamma}{\partial \tau^{2}}-\frac{1}{2}(\nabla f f+\varepsilon \nabla(\nabla \cdot f))(\gamma)+\sqrt{2 \varepsilon} \frac{\partial W}{\partial s} & \forall(\tau, s) \in[0,1] \times(0, \infty) \\
\Phi(\gamma)=\xi_{A},\left(\frac{\partial \gamma}{\partial s}\right)^{\|}=(2 \varepsilon \operatorname{Sn}(\gamma)-f(\gamma))^{\|} & \forall(\tau, t) \in\{0\} \times(0, \infty) \\
\Phi(\gamma)=\xi_{B},\left(\frac{\partial \gamma}{\partial s}\right)^{\|}=(f(\gamma)-2 \varepsilon \operatorname{Sn}(\gamma))^{\|} & \forall(\tau, t) \in\{1\} \times(0, \infty) \\
\gamma=\gamma_{0} & \forall(\tau, s) \in[0,1] \times\{0\}
\end{array}
$$

where $\partial W / \partial s$ is space-time white noise and we have introduced the various shorthands: $n=\nabla \Phi /|\nabla \Phi|$ for the unit normal to the level sets $\{\Phi(x)=\xi\}, f^{\|}=(I-n \otimes n) f$ for the vector field $f$ tangent to the level sets, and $S=\nabla^{2} \Phi /|\nabla \Phi|$ for the shape operator (second fundamental form) of $\{\Phi(x)=\xi\}$ understood as a submanifold of $\mathscr{X}$.

Note that although $\gamma$ lives in $\mathscr{X} \subseteq \mathbb{R}^{d}$, which may be high-dimensional, its two arguments are scalar variables (namely, arc length $\tau$ and time $s$ ). Methods for numerically solving SPDEs such as (8) are discussed in, e.g., [6].

\section{REFERENCES}

1. C. Jarzynski, Phys. Rev. Lett. 78, 2690-2693 (1996).

2. G. Crooks, J. Stat. Phys. 90, 1481-1487 (1998).

3. J.C. Latorre, C. Hartmann, and Ch. Schütte, Procedia Computer Science 1, 1591-1600 (2010).

4. G. Ciccotti, T. Lelièvre, and E. Vanden-Eijnden, Commun. Pure Appl. Math. 61, 371-408 (2008).

5. A. M. Stuart, J. Voss, and P. Wiberg, Commun. Math. Sci. 2, 685-697 (2004).

6. A. Beskos, G. O. Roberts, A.M. Stuart, and J. Voss, Stochastics and Dynamics 8, 319-350 (2008). 
Copyright of AIP Conference Proceedings is the property of American Institute of Physics and its content may not be copied or emailed to multiple sites or posted to a listserv without the copyright holder's express written permission. However, users may print, download, or email articles for individual use. 